\title{
Impact of RIN on Optical CDMA Transmission System
}

\author{
Mudavath Raju \\ Electronics \& Communication \\ Engineering \\ National Institute of Technology \\ Hamirpur, India
}

\author{
Naresh Kumar \\ Electronics \& Communication \\ Engineering \\ Shoolini University Solan, India
}

\author{
D.R.Rana \\ Electronics \& Communication \\ Engineering \\ National Institute of Technology \\ Hamirpur, India
}

\begin{abstract}
Optical code division multiple access (OCDMA) is one of the most upcoming technologies for future multiple access techniques along with WDMA and OTDMA. Optical CDMA offers greater scalability than other optical multiplexing technique and it provides flexible quality of service and data access security. OCDMA requires increasing demand for high speed, large capacity communications in optical networks which allow multiple users to share the fiber bandwidth. The data for multiple users are multiplexed asynchronously. In this Paper, we have presented an OCDMA model to the range of $90 \mathrm{~km}$. The simulation results reveal that the transmission distance is limited mainly by the Relative Intensity Noise (RIN) which arises when there is instability in the power level of a laser in the system because of the fact that RIN can be generated from fluctuations in the laser gain medium.
\end{abstract}

\section{General Terms}

Optical Code Division Multiple Access, Relative Intensity Noise.

\section{Keywords}

Optical Code Division Multiple Access (OCDMA), Bit Error Rate (BER), Pseudo Orthogonal (PSO) Code, Relative Intensity Noise (RIN), Non-Return-to-Zero (NRZ), Single Mode fiber (SMF).

\section{INTRODUCTION}

Since the late 1980s, there have been steady advancement in the field of coding-based optical systems and networks by optical code-division multiple accesses (O-CDMA)[1].The increasing demands for higher speed and advanced services in access networks require a bandwidth of above $50 \mathrm{Mbps}$ for next-generation services to end users. The use of technologies based on optical fibers can easily achieve bandwidths higher than $100 \mathrm{Mbps}$ and at the same time can reduce maintenance and repair costs [2-3]. OCDMA operates asynchronously without centralized control and does not suffer from packet collisions. As a result, OCDMA systems have lower latencies than TDMA. In an OCDMA system each bit is divided into $\mathrm{N}$ time periods called chips. The asynchronous data transmission can simplify network management and control. Particularly, OCDMA can provide a secure network connection providing dynamic encoding [4]. Relative intensity noise (RIN) is the instability in the power level of a laser. The noise term is important to describe lasers used in fiber-optic communication and it is usually the dominant source of bit error rate in an OCDMA. RIN can be generated from cavity vibration, fluctuations in the laser gain medium [5]. The RIN has been a concern since the early beginning of laser optics. Generally, the RIN is measured as the DC fluctuation of the current at the output of a photo detector [6]. For high performance measurement system like in high speed optical telecommunications for which many parameters influence its performance, source noise must be estimated and measured.
Relative Intensity Noise is totally negligible for frequencies higher than $10 \mathrm{MHz}$ and measure RIN at this frequency by using another reference laser must be used. The laser is a DFB laser. Its frequency is stabilized thanks to a gas cell and its RIN is estimated lower than $-160 \mathrm{~dB} / \mathrm{Hz}$ [7]. Another use of OCDMA in optical communications for increasing number of users is studied and multiple access interference is the dominant factor limiting the user capacity of the system. An Optical Hard Limiter (OHL) placed at the front end of the receiver can reduce the effects of the MAI. But it can't handle the random signals effectively [8]. The MAI in optical CDMA which degraded the efficiency of system by increasing bit error rate. Use of spectral phase encoding O-CDMA system reduced the MAI as seen in the bit error rate performance [9]. OCDMA systems suffer from MAI as the main performance limiting factor and different types of receiver structures have been proposed to remedy this limitation [10-14]. Future optical CDMA networks need to support multimedia services. Research is still going on to develop a better OCDMA code which will support the multimedia services. The design of a set of eight matrix codes for operation at $2.5 \mathrm{Gbps}$ has been described [15]. The intensity fluctuations directly affect on the performance of lightwave systems and it is characterized by the relative intensity noise (RIN) which is discussed. The design of OCDMA system with improved coding techniques are presented, which helps in reducing hardware requirement and still offers better performance for higher number of users with low noise [16]. This paper work is focused on the impact of different RIN value on optical OCDMA system. Here we propose the simulative OCDMA transmitter and receiver with optical fibre reported in section 2 . System description is discussed in section 3.The simulation results have been discussed in section 4 . The conclusion of our simulation results is presented in section 5 .

\section{SYSTEM DESCRIPTION}

In the proposed optical CDMA transmission link (Fig. 1.), $2.50 \mathrm{Gbps}$ data signal is generated with NRZ modulation. The $2.50 \mathrm{Gbps}$ NRZ data signal is then modulated by means of MZM modulator and then transmitted over SM fiber. Four mode-lock lasers are used to create a dense WDM multifrequency light source of $3 \mathrm{~mW}$ operating at 1550.0-1551.2 $\mathrm{nm}$. Encoded optical CDMA signal is transmitted over SM fibre having an attenuation of $0.2 \mathrm{~dB} / \mathrm{Km}$. Here we are using sixteen OC-48 users requiring sixteen distinct signature codes. For this purpose Pseudo Orthogonal (PSO) matrix codes [9] are used as they are popular for OCDMA applications primarily because they retain the correlation advantages of PSO linear sequences while reducing the need for bandwidth expansion. PSO matrix codes also generate a larger code set. An interesting variation is described in [8] where some of the wavelength/time (W/T) matrix codes can permit extensive wavelength reuse and some can allow extensive time-slot reuse. In this model, an extensive time-slot reuse sequence is used for User $1(\lambda 1 \lambda 3 ; 0 ; \lambda 2 \lambda 4 ; 0)$. 


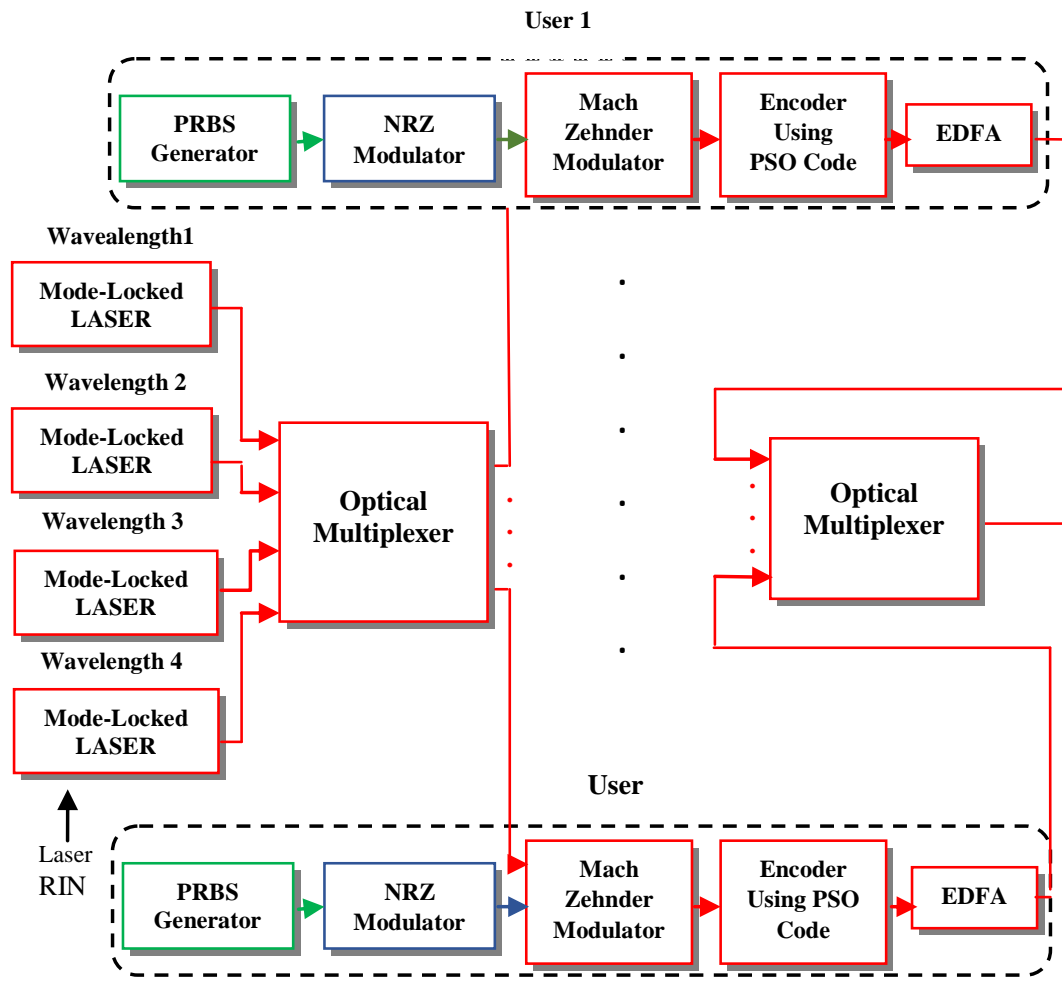

Optical CDMA Transmitter
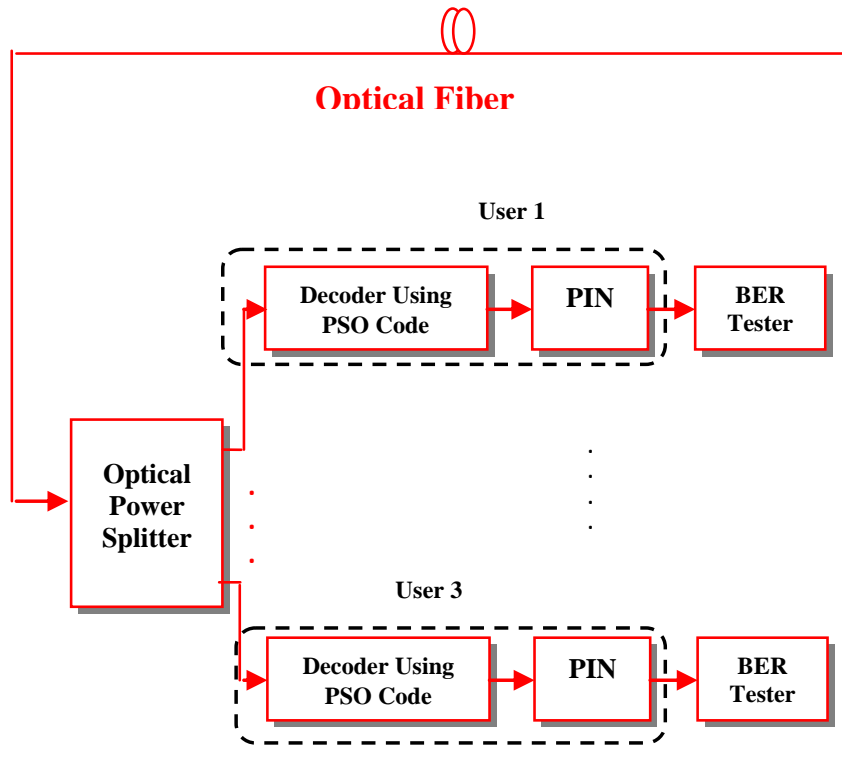

Optical CDMA Receiver

Figure 1: Simulation setup of optical CDMA transmission link

There are four time slots used without any guard band giving the chip period of $100 \mathrm{ps}$. As the receiver information is retrieved by a decoder using the same PSO code and then the optical signal is converted back into electrical form through a PIN photo diode and hence $2.50 \mathrm{Gbps}$ optical CDMA data is recovered successfully.

\section{RESULTS AND DISCUSSION}

Two cases of performance analysis are: Case I investigates different parameters on optical CDMA transmission link and
Case II gives impact of RIN in optical CDMA transmission link.

\section{Case I: Performance Investigations of} Different parameter in optical CDMA transmission link

The parameters used in this case are data rate $2.50 \mathrm{Gbps}$, aperture area $180 \mathrm{~cm}^{2}$, transmitted power $3 \mathrm{~mW}$, fiber length $90 \mathrm{~km}$, Sigma Add=1.9, Divergence Angle $=0.25 \mathrm{mrad}$.

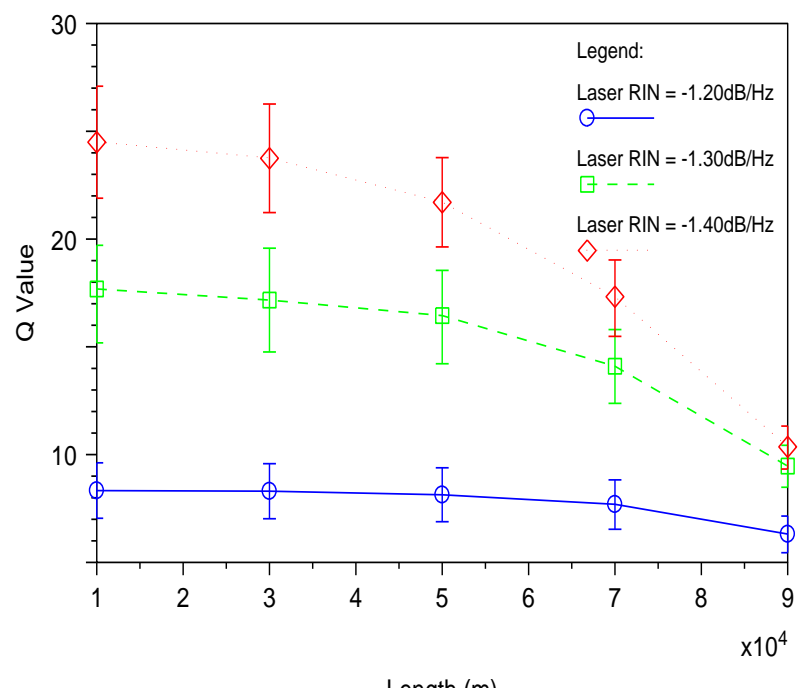

Length (m)

(a)

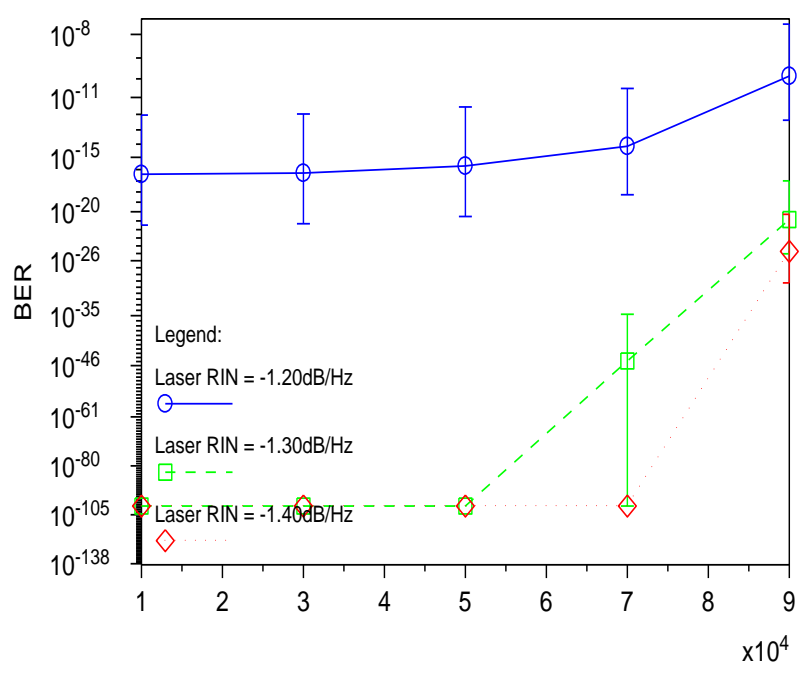

(b)

Fig. 2: (a) Evaluation of $Q$ Value versus transmission length with different input laser RIN levels for single user, (b) Evaluation of BER versus transmission length with different input Laser RIN levels for single user.

The graphs of Q-value and BER versus transmission length at different transmitter laser RIN level are presented in figure 2 (a) \& (b). From results it has been observed that there is significant decrease in the value of $\mathrm{Q}$ factor, which lies within [6.8, 6.5 and 4], [17.5, $16 \& 9]$ and [24.5, $22 \& 11]$ for transmission distance of $10 \mathrm{~km}, 50 \mathrm{~km}$ and $90 \mathrm{~km}$ in case of laser RIN $-1.20 \mathrm{~dB} / \mathrm{Hz}, \quad-1.30 \mathrm{~dB} / \mathrm{Hz}$ and $-1.40 \mathrm{~dB} / \mathrm{Hz}$ respectively. In case of BER, From results it has been 
observed that there is significant increase in the value of BER, which lies within $\left[10^{-16.5}, 10^{-16} \& 10^{-10.5}\right],\left[10^{-100}, 10^{-100} \& 10^{-}\right.$ $\left.{ }^{24}\right]$ and $\left[10^{-100}, 10^{-100} \& 10^{-25.5}\right]$ for a transmission distance of $10 \mathrm{~km}, 50 \mathrm{~km}$ and $90 \mathrm{~km}$ in case of laser RIN $-1.20 \mathrm{~dB} / \mathrm{Hz}$, $1.30 \mathrm{~dB} / \mathrm{Hz}$ and $-1.40 \mathrm{~dB} / \mathrm{Hz}$ of transmitter laser RIN respectively.

\section{Case II: Impact of Laser RIN in optical CDMA transmission link}

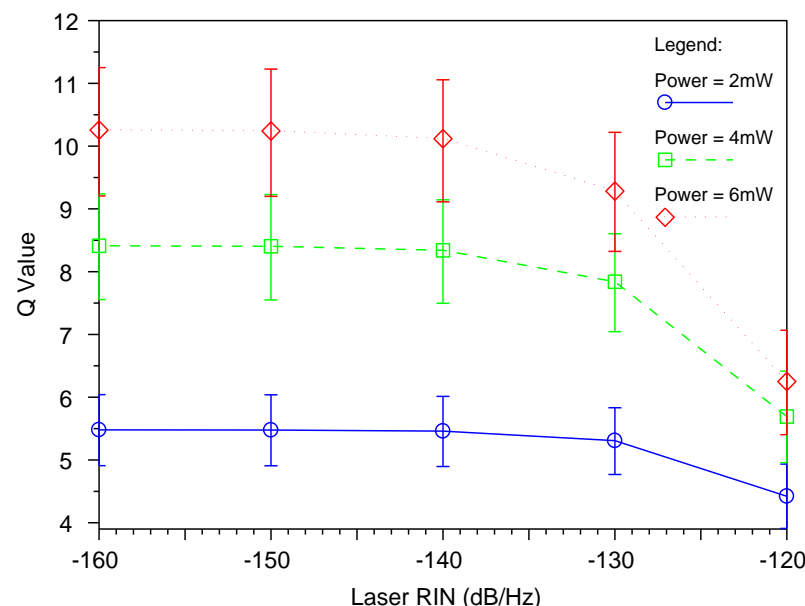

(a)

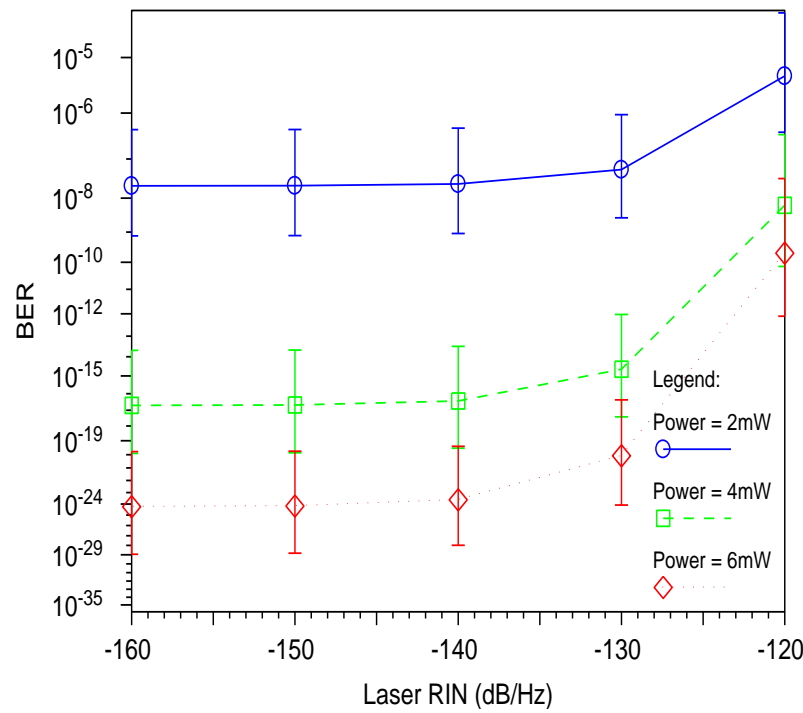

(b)

Fig. 3: (a) Evaluation of $Q$ Value versus laser RIN with different power levels for single user, (b) Evaluation of BER versus laser RIN with different power levels for single user.

The graphs of $\mathrm{Q}$ value and BER versus transmission laser RIN $(\mathrm{dB} / \mathrm{Hz})$ are given in figure $3(\mathrm{a}) \& 3(\mathrm{~b})$. From results it has been observed that there is significant decrease in the value of $\mathrm{Q}$ factor, which lies within 5.5 to $4.5,8.4$ to 5.5 and 10.4 to 6.3 for a transmission laser RIN of $-160 \mathrm{~dB} / \mathrm{Hz}$ to $-120 \mathrm{~dB} / \mathrm{Hz}$ in case of transmitter power $2 \mathrm{~mW}, 4 \mathrm{~mW}$ and $6 \mathrm{~mW}$. In case of BER, From results it has been observed that there is significant increase in the value of BER, which lies within $10^{-}$ 7.8 to $10^{-5.5}, 10^{-16.5}$ to $10^{-8.5}$ and $10^{-24}$ to $10^{-9.5}$ for a transmission laser RIN of $-160 \mathrm{~dB} / \mathrm{Hz}$ to $-120 \mathrm{~dB} / \mathrm{Hz}$ in case of transmitter power $2 \mathrm{~mW}, 4 \mathrm{~mW}$ and $6 \mathrm{~mW}$.

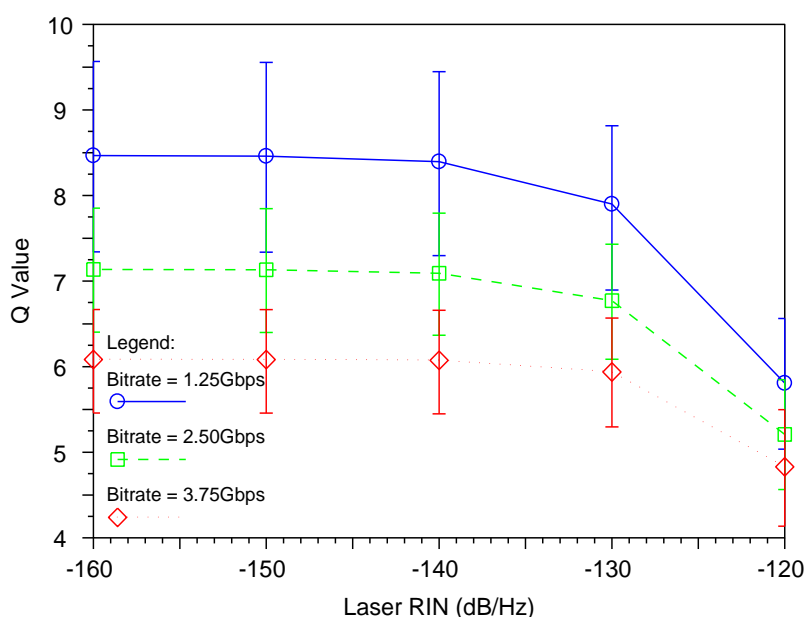

(a)

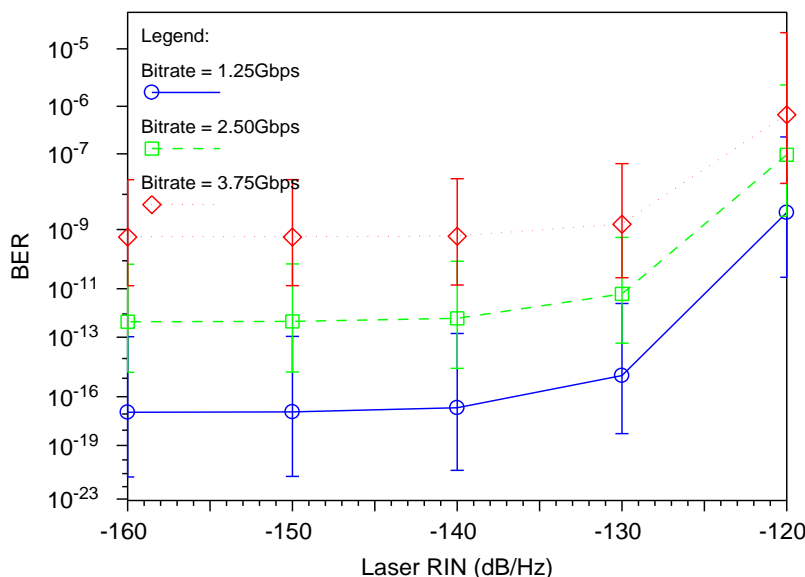

(b)

Fig. 4: (a) Evaluation of Q Value versus Laser RIN with different input bit rate for single user, (b) Evaluation of BER versus Laser RIN with different input bit rate for single user

The graphs of Q-value and BER versus transmission laser RIN at different bitrates are presented in figure 4 (a) \& (b). From results it has been observed that there is significant decrease in the value of $\mathrm{Q}$ factor, which lies within $[8.4,8.3$ \& 5.8], [7.1, $7 \& 5.2]$ and [6.1, $6 \& 4.9]$ for transmission laser RIN of $-160 \mathrm{~dB} / \mathrm{Hz},-140 \mathrm{~dB} / \mathrm{Hz}$ and $-120 \mathrm{~dB} / \mathrm{Hz}$ in case of bitrates $1.25 \mathrm{Gbps}, 2.50 \mathrm{Gbps}$ and $3.75 \mathrm{Gbps}$ respectively. In case of BER, From results it has been observed that there is significant increase in the value of BER, which lies within $\left[10^{-17}, 10^{-16}\right.$ and $\left.10^{-8.5}\right],\left[10^{-12.4}, 10^{-12}\right.$ and $\left.10^{-7}\right]$ and $\left[10^{-9.4}, 10^{-9}\right.$ $\& 10^{-6.5}$ ] for transmission laser RIN from $-160 \mathrm{~dB} / \mathrm{Hz},-140$ $\mathrm{dB} / \mathrm{Hz}$ and $-120 \mathrm{~dB} / \mathrm{Hz}$ in case of bitrates $1.25 \mathrm{Gbps}, 2.50$ Gbps and $3.75 \mathrm{Gbps}$ respectively. 


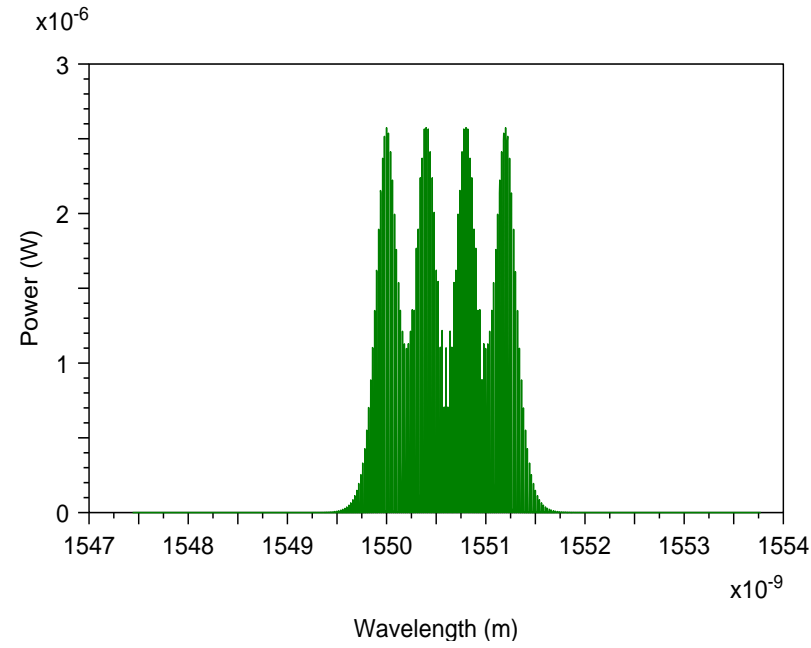

Figure 5: Eye diagram of signal After WDM

The eye diagrams for four wavelengths $(\mathrm{nm})$ are shown in figure 5. It has been observed that the efficiency of the system is transmitting number of wavelength. Thus it is established that laser RIN plays key detrimental role in optical CDMA communication system.

\section{CONCLUSION}

The design of an optical CDMA transmission system at data rate of $2.5 \mathrm{Gbps}$ for $90 \mathrm{~km}$ length is presented. Performance investigation on this designed optical CDMA was carried out using with different parameters and laser RIN. It is concluded that impact of laser RIN on OCDMA is because of fluctuations of laser light. Further, transmission range also increases with the increase in transmitter power. However the efficiency of OCDMA is degraded with the increase of bit rates. It is also concluded that the efficiency of OCDMA is degraded by decreasing laser $\mathrm{RIN}$ in $\mathrm{dB} / \mathrm{Hz}$.

\section{REFERENCES}

[1] Yen-Feng Li, Guu-Chang Yang "Extended Reed-Solomon Codes for Optical CDMA" IEEE Transactions on Communications, Vol. 61, No. 6, June 2013.

[2] Yin, H., Richardson, D. J. 2007. Optical Code Division Multiple Access Communication Networks Theory and Applications, Tsinghua University Press, Beijing and Springer-Verlag GmbH Berlin Heidelberg.

[3] Ahmad, S. 2010 what is Optical CDMA. http:// ezinearticles.com/?What-is-OpticalCDMA?\&id= 4177189.

[4] M. Yamada, N. Takeuchi, K. Sakumoto, and Y. Kuwamura, "Variation of Relative Intensity Noise With Optical Power in InGaAsP Semiconductor Optical Amplifier" IEEE Photonics Technology Letters, Vol. 24, No. 22, November 15, 2012

[5] R. Zhang and Frank G. Shi, "Manufacturing of Laser Diode Modules: Integration and Automation of Laser
Diode-Fiber Alignment and RIN Characterization" IEEE Transactions on Advanced Packaging, Vol. 26, No. 2, May 2003

[6] Enrico Rubiola, And Laurent Larger "Measurement of the Laser Relative Intensity Noise" 978-1-4244-35104/09/2009 IEEE

[7] J. Poette, P. Besnard, L. Bramerie and Jean-Claude Simon "Highly-Sensitive Measurement Technique of Relative Intensity Noise and Laser Characterization" Noise and Fluctuations in Photonics, Quantum Optics, and Communications Edited By Leon Cohen, Proc. Of SPIE Vol. 6603, 66031R, (2007)

[8] John. A and Raj S.S, "Reduction of MAI using Optical Normaliser in an Optical CDMA system", Third International Conference on Advances in Computing and Communications, IEEE Conference Publications, 2013.

[9] Bhosale S.R, Nalbalwar S.L, Deosarkar S.B, “ Design and Performance Analysis of Eight User Spectral Phase Encoding O-CDMA time domain System at Different bit rate for MANET", Advanced Communication Technology (ICACT), 14th International Conference on, IEEE Conference Publications, 2012.

[10]T.Ohtsuki, "Channel interference cancellation using electro optic switch and optical hardlimiters for directdetection optical CDMA systems," IEEE J. Lightw. Technol., vol. 16, no. 4, pp. 520-526, Apr.1998.

[11] M. H. M. Shalaby, "Chip-level detection in optical code division multiple access," IEEE J. Lightw. Technol., vol. 16, no. 6, pp. 1077-1087, Jun. 1998.

[12]S. Mashhadi and J. A. Salehi, "Code-division multipleaccess techniques in optical fiber networks-Part III: Optical AND gate receiver structure using generalized optical orthogonal codes," IEEE Trans.Commun., vol. 54, pp. 1457-1468, Aug. 2006.

[13]B. M. Ghaffari and J. A. Salehi, "Multiclass, multistage, and multilevel fiber-optic CDMA signaling techniques based on advanced binary optical logic gate elements," IEEE Trans. Commun., vol. 57, pp. 1424-1432, May 2009.

[14]H. Beyranvand, B. M. Ghaffari, and J. A. Salehi, "Multirate, differentiated-QoS, and multilevel fiber-optic CDMA system via optical logic gate elements," IEEE J. Lightw. Technol., vol. 27, pp. 4348-4359, Oct.2009.

[15]Antonio J. Mendez, R.M. Gagliardi, Vincent J. Hernandez, "Design and Performance Analysis of Wavelength/Time (W/T) Matrix Codes for Optical CDMA" IEEE Journal Of Lightwave Technology, Vol. 21, No. 11, November 2003.

[16]H. K. Hisham, A. F. Abas, Ghafour A. Mahdiraji "Relative Intensity Noise Reduction By Optimizing Fiber Grating Fabry-Perot Laser Parameters" IEEE Journal Of Quantum Electronics, Vol. 48, No. 3, March 2012. 